\title{
Effect of explosive thermal evaporation conditions on the phase composition, crystallite orientation, electrical and magnetic properties of heteroepitaxial InSb films on semi- insulating GaAs (100)
}

\author{
V. V. Uglov ${ }^{1)}$, A. P. Drapezo ${ }^{2)}$, A. K. Kuleshov ${ }^{\text {I) }}$, D. P. Rusalsky ${ }^{1)}$, E. A. Kolesnikoval) \\ ${ }^{1)}$ Belarusian State University, 4 Nezavisimosty ave., Minsk, 220030, Belarus \\ tel. +375172096257, e-mail: kuleshak@bsu.by \\ ${ }^{2)}$ LLC «Scientific and technical center Vist group sensor», 2B Novatorskaya str., off. 204, \\ Minsk, 220053, Belarus
}

\begin{abstract}
In present work the explosive thermal evaporation was used to form heteroepitaxial InSb films on GaAs (100) substrates. The investigation results of phase composition, orientation of crystallites, electrical and magnetic properties are presented. It is shown that heteroepitaxial InSb films form in the deposition temperature range of $375-410{ }^{\circ} \mathrm{C}$. At lower deposition temperatures, InSb films have a polycrystalline structure. The obtained heteroepitaxial $\mathrm{InSb}$ films have high values of the Hall potential (more than $0.5-0.6 \mathrm{~V}$ ), the values of the Hall mobility of the charge carriers are in the range of $(16-19) \times 10^{3} \mathrm{~cm}^{2} /(\mathrm{V} \times \mathrm{s})$.

Key words: indium antimonide, gallium arsenide, thin films, vacuum deposition, structure, electrical properties, magnetic properties.
\end{abstract}

\section{Introduction}


Indium antimonide $(\mathrm{InSb})$ is a narrow-band straight-band semiconductor of the $\mathrm{A}^{\mathrm{III}} \mathrm{B}^{\mathrm{V}}$ group with the smallest energy gap of $0.17 \mathrm{eV}$ at room temperature. Due to its properties, InSb is widely used in the field of micro- and optoelectronics. InSb has a record high electron mobility and free path length, as well as operating temperature range over 500 degrees. The photoconductivity of InSb lies in the IR range with a wavelength up to $\lambda=7 \mu \mathrm{m}$. That is why $\mathrm{InSb}$ is used for manufacturing of highly sensitive solar cells, Hall sensors, magnetoresistors, optical filters (Balvanovich, 1981).

In modern microelectronics, the miniaturization of components and elements of various devices, including based on InSb, comes to the fore in relation to single-crystal "large" devices. Modern methods of epitaxial growth make it possible to impart the properties of single crystals to hetero and homoepitaxial film structures deposited on specially prepared crystal substrates. The use of such film structures makes it possible to create modern devices of micro-electron version. As a result, the study of epitaxial InSb films deposited on crystal substrates is of great practical interest for the manufacture of miniature highly sensitive Hall convertor used for sensors of physical quantities: rotation angle, pressure, linear displacement, etc. (Balvanovich, 1981; Bennett et al., 2005).

The most used crystalline material for epitaxial growth of InSb (lattice parameter $6.48 \AA$ ) is i-GaAs (lattice parameter $5.65 \AA$ ) (Balvanovich, 1981). However, lattice mismatch between InSb and GaAs is large and it makes the growth of epitaxial InSb film on GaAs very difficult process. Therefore, the formation of smooth epitaxial perfect InSb films is a complex scientific and technological task (Balvanovich, 1981). At the moment, the exact mechanisms and models of epitaxial growth of InSb on GaAs depending on the deposition conditions are not established. The most widely used technique for the deposition of InSb films is molecular beam epitaxy 
(MBE). This method provides high quality of the films and makes it possible to obtain smooth, defect-free surfaces. The low growth rate and relatively low growth temperature characterize MBE in comparison with other technologies used for thin films and multilayer structures synthesis (Joyce et al, 1989). The MBE-formation and investigation of the properties of InSb films on GaAs substrates are discussed in (Zhang et al., 2004; Okamoto and Shibasaki, 2003). Electron beam evaporation (EBPVD) is also used to produce InSb films. The EBPVD-deposition of InSb films on glass substrate at temperature of $30-100{ }^{\circ} \mathrm{C}$ with a thickness of $300-400 \mathrm{~nm}$ and its structural state and electrical properties is discussed in (Rahul et al., 2011). In particular, on the base of X-ray diffraction (XRD) studies the authors found that the resulting films have a polycrystalline structure and are oriented along the planes (111) and (220). At the same time, the degree of crystallinity of the films increases significantly with an increase in the substrate temperature.

The more technologically advanced technique which uses inexpensive vacuum equipment that allows in a short time to obtain InSb films including epitaxial films in a wide thickness range of from fractions to tens microns is explosive thermal evaporation. The essence of the method consists in the evaporation in a vacuum of powders of compound elements placed on evaporator through the stage of evaporation of the light-volatile component and enrichment with the hardvolatile component. At the final stage of evaporation, only the hard-flowing component gets into the steam. If a large number of small particles are evaporated, each of which is at a different stage of evaporation, then with a uniform distribution of their evaporation moments over time, the vapor composition corresponding to the composition of the compound is provided. The epitaxial growth of InSb thin films on GaAs during explosive thermal evaporation is a rather complicated process, depending on the substrate temperature, the temperature of the powder 
evaporator, the purity of the powder, the particle size and the speed of supply of the powder into the evaporator.

The influence of deposition parameters (deposition temperature, thickness) during explosive thermal evaporation on the electrical and magnetic characteristics of InSb films deposited on undirected substrates and GaAs single-crystal plates was studied in (Oszwaldowskia et al., 2004; Oszwaldowskia and Berus, 2006). In these works, it is reported that with a film thickness of less than $0.3 \mu \mathrm{m}$, the mobility and concentration of carriers are small. The increase of thickness of the films up to about 1 micrometer resulted in increase of carrier concentration in such samples up to $4 \times 10^{17} \mathrm{~cm}^{-3}$. The further increase in the thickness of the films up to $4 \mu \mathrm{m}$ does not significantly change the electrical and magnetic properties of the films. In (Oszwaldowskia et al., 2004), the authors investigated the growth of InSb films on undirected substrates, the temperature of which varied from 160 to $450{ }^{\circ} \mathrm{C}$. At a substrate temperature of less than $400{ }^{\circ} \mathrm{C}$, the films have a prevalent orientation in the (110) plane. At a substrate temperature greater than $400{ }^{\circ} \mathrm{C}$, the orientations of (110) and (111) become preferred, while at higher temperatures the (111) orientation becomes preferred. The presence of preferential orientations in the film at a substrate temperature of more than $400{ }^{\circ} \mathrm{C}$ is associated with the fact that the planes (110) and (111) have the lowest values of the surface energy of their formation.

The influence of different types of GaAs substrates orientation on the processes of $\mathrm{InSb}$ deposition is presented in (Debnath et al., 2004; Kimukin et al., 2003). The most successful regime was the two-stage MBE process of $\mathrm{InSb}$ applying at temperature of $320-420{ }^{\circ} \mathrm{C}$ which made it possible to create epitaxial InSb films on (111) GaAs of sufficient perfection, according to the results of measurements of their electrical properties. For InSb films on GaAs (100), the application of the above-mentioned deposition temperature ranges did not allow the creation of 
perfect epitaxial films. There is also evidence of a significant decrease in the crystallinity of InSb films at deposition temperatures below $340{ }^{\circ} \mathrm{C}$. Thus, the substrate temperature is the most important parameter that determines the condensation mechanism, the form of growth, the phase and structural uniformity and the epitaxial perfection of thin films. It should be noted that in this works the electron backscatter diffraction (EBSD) was not used for studding of orientation of micro-crystallites in films as a function of the deposition temperature.

The aim of this work is to find the regularities of the influence of the substrate temperature $\left(350-420^{\circ} \mathrm{C}\right)$ during explosive thermal evaporation deposition on the phase composition, orientation of crystallites, electrical and magnetic properties of InSb films on GaAs (100) by XRD, EBSD, measurements of the electrical and magnetic properties of the films.

\section{Materials and experimental details}

InSb films on semi-insulating single-crystal GaAs (100) substrates deposited by explosive thermal evaporation of a single-crystal powder were investigated. The procedure of explosive thermal evaporation is following. The powdered material from the vibrating feeder device is continuously supplied onto the evaporator where it is heated to a temperature that allows instant evaporation of particles of material, followed by condensation on the substrate surface (Maissel and Glang, 1977). InSb powder with a carrier concentration of $n=10^{15}-10^{16}$ $\mathrm{cm}^{-3}$ was used for film deposition. The films were deposited in vacuum through masks to give the sample a rectangular shape with contact pads. Fig. 1 displays the main elements of the vacuum equipment for carrying out the process of explosive thermal evaporation. 


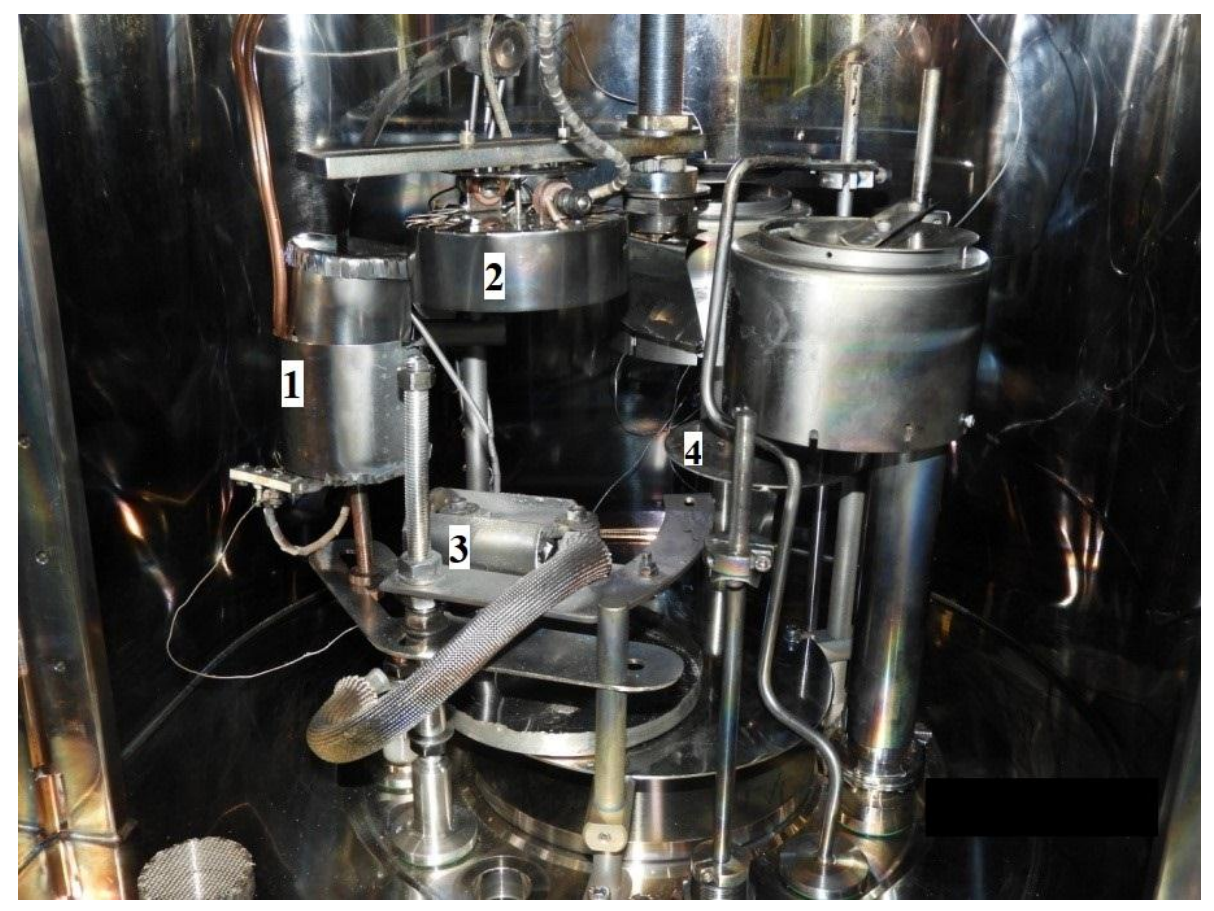

Figure 1. General view the main elements of the vacuum equipment for carrying out the process of explosive thermal evaporation: 1 - vibrating feeder device, 2 - substrate-sample holder with heater, 3 - evaporator, 4 damper of evaporator

The temperature of the substrate holder with the GaAs plate was varied by means of a heater in the range of $350-420{ }^{\circ} \mathrm{C}$. During the experiment, the temperature of the heater is controlled by a thermocouple. Also, during the deposition, the thickness and the deposition rate of the film was monitored by a quartz sensor. The thickness of formed film was also measured using an MII-4 interferometer and a profilometer. The measured film thickness range was $1.1-$ $3.2 \mu \mathrm{m}$.

Crystal state of the obtained films was investigated by the XRD analysis with $\mathrm{CuK}_{\alpha}$ radiation using a Rigaku Ultima IV diffractometer. The orientation of the microcrystallites of the deposited films was analyzed by means the EBSD on a scanning electron microscope (SEM) using a LEO1455VP one. 
Measurements of the electrical and magnetic properties of the obtained rectangular films with contact pads included the measurement of the voltage and Hall voltage at a given value of the magnetic field and the electric current flowing through the sample (Kuchis, 1974). The magnetic field was created by the electromagnet. In this investigation, the magnetic induction value was $0.44 \mathrm{~T}$. The values of the concentration $(n)$ and mobility $(\mu)$ of charge carriers in the studied films were calculated from the following equations:

$$
n=\frac{I B}{d U_{x} e}
$$

where $I$ is the electric current, $B$ is the magnetic induction, $U_{x}$ is the Hall voltage, $e$ is the elementary charge and $d$ is the thickness film.

$$
\mu=\frac{U_{x} l}{B b U},
$$

where $l$ is the length film, $U$ is the measured voltage and $b$ is the width film.

\section{Result and discussions}

The results of the XRD analysis of the phase composition of films on semi-insulating GaAs (100) formed by explosive thermal evaporation of InSb powder as a function of the substrate temperature in the temperature range of $350-375{ }^{\circ} \mathrm{C}$ are displays in Fig. 2. 


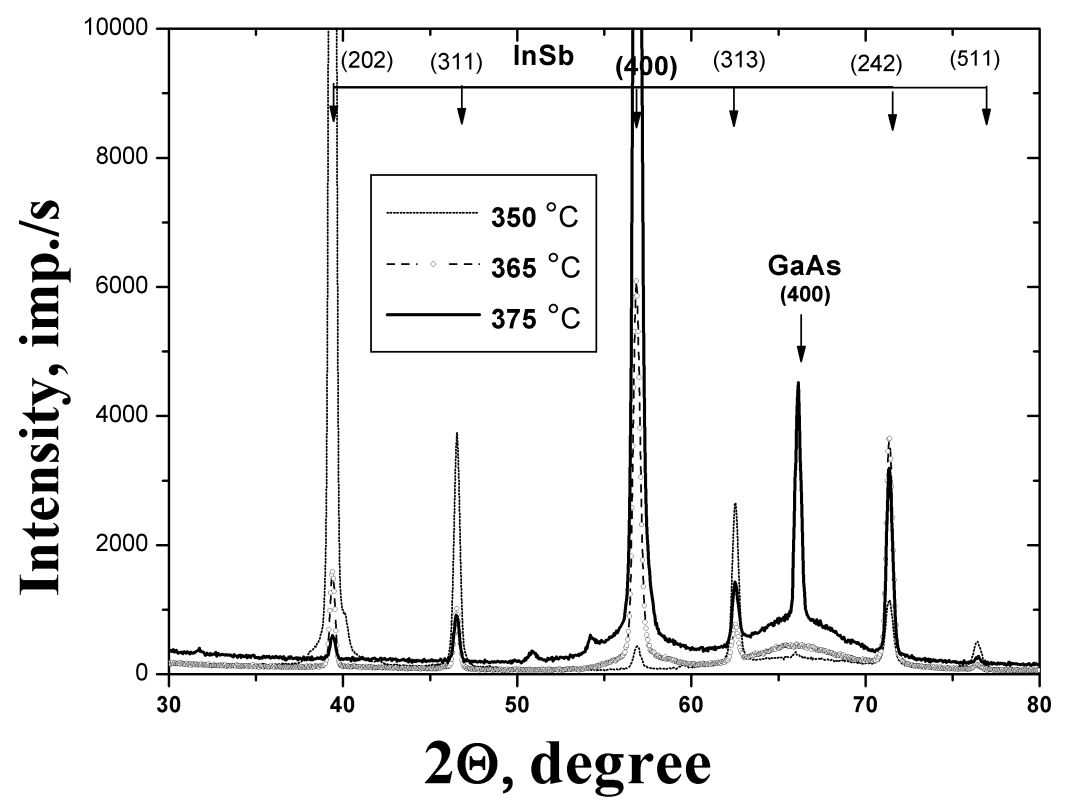

Figure 2. XRD patterns of film samples deposited on semi-insulating GaAs plates by explosive thermal evaporation of InSb powder as a function of the substrate temperature

From the XRD patterns presented in Fig. 2 and their analysis using the ICDD-PDF 2 database, it follows that at the deposition temperature of $350{ }^{\circ} \mathrm{C}$, the phase composition of the film is an InSb compound. This film is characterized by the prevalent (101) orientation of the crystallites. When the film deposition temperature increases to $365^{\circ} \mathrm{C}$, the prevalent orientation of the film crystallites becomes (100) which crystallographically corresponds to GaAs (100) single crystal substrate. Diffraction reflections from other orientations of crystallites of the InSb compound are also presented in the diffraction pattern (Fig. 2). Consequently, the InSb film deposited at a temperature of $365^{\circ} \mathrm{C}$ is polycrystalline. The intensity of diffraction (400) line becomes the largest at a deposition temperature of $375^{\circ} \mathrm{C}$. The ratio of the intensity of the diffraction (400) line to the (313) line, which has the next intensity of all InSb lines, exceeds 30. 
The InSb film deposited on a single-crystal GaAs substrate with orientation (100) repeats this orientation, i.e. it is a heteroepitaxial $\mathrm{InSb}$ film grown on GaAs with orientation (100).

For a detailed investigation of the orientation of the crystallites in the surface layers of the InSb film as a function of the deposition temperature, the EBSD on a SEM was also used. Fig. 3 displays the results of this investigation.

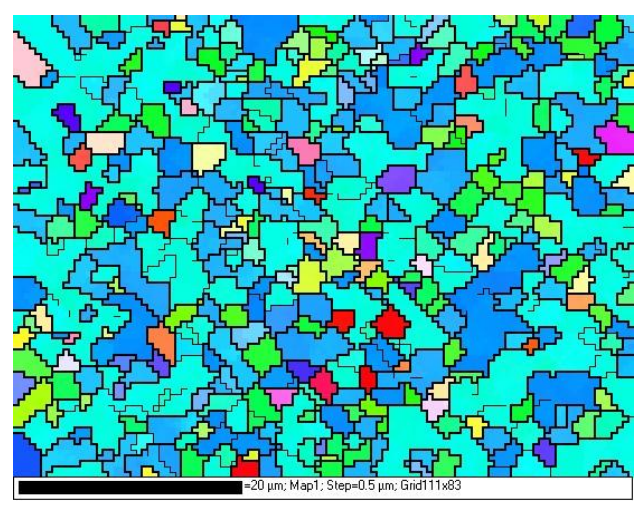

a

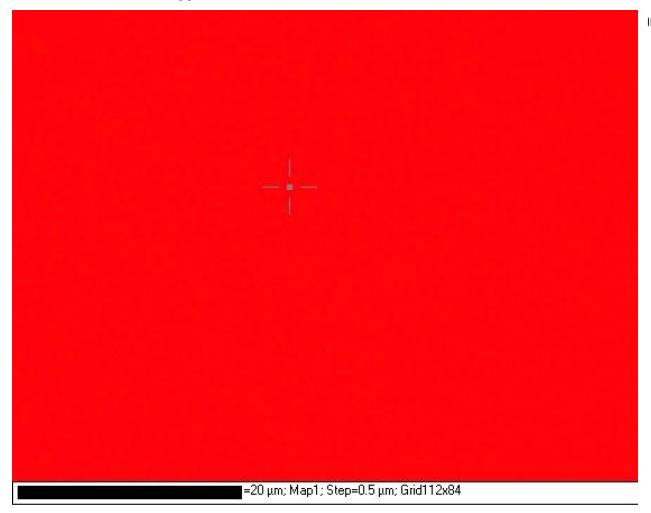

$\mathrm{c}$

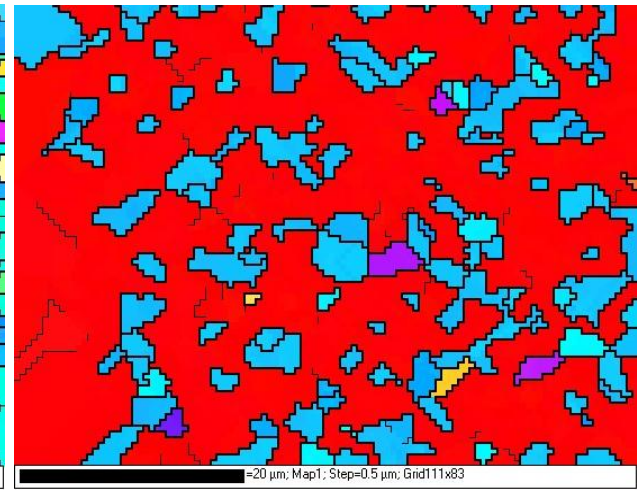

b

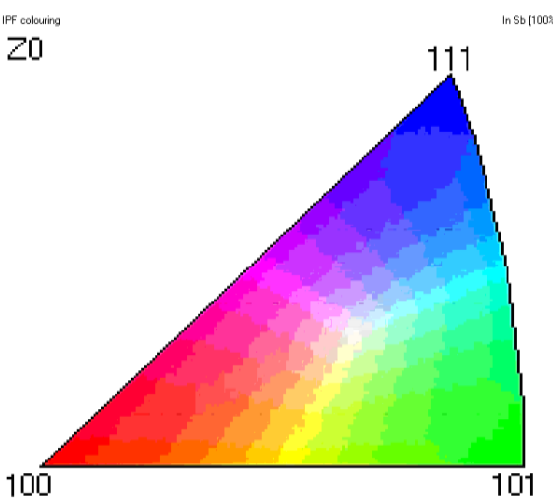

$\mathrm{d}$

Figure 3. Scanning images of the orientation of crystallites of a section of an InSb film obtained by explosive thermal evaporation at deposition temperatures: $a-350^{\circ} \mathrm{C} ; \mathrm{b}-365^{\circ} \mathrm{C} ; \mathrm{c}-375^{\circ} \mathrm{C}$; $d$ - map of the correspondence of the color image and the orientation of crystallites for the InSb film in EBSD

From the data presented in Fig. 3 it follows that in the surface layer of the film at the deposition temperature of $350^{\circ} \mathrm{C}$ the orientations of the crystallites of the film are close to (101), 
(111). When the deposition temperature increases to $365^{\circ} \mathrm{C}$, the orientation (100) of the crystallites becomes prevalent. At a deposition temperature of $375{ }^{\circ} \mathrm{C}$, there is only one crystallite orientation (100) in the surface layer of the film. It should be noted that the results of investigation of the structure and texture of InSb films deposited at a temperature of $370{ }^{\circ} \mathrm{C}$ are identical to the above data for a film deposited at a temperature of $375^{\circ} \mathrm{C}$. Thus, the results of XRD and EBSD investigations (Figs. 2, 3) of the crystallite orientations in InSb films in the deposition temperature range of $350-375^{\circ} \mathrm{C}$ are in good agreements with each others.

The XRD patterns of the film deposited at $410{ }^{\circ} \mathrm{C}$ (Fig. 4) is similar to the XRD patterns of the film deposited at $375{ }^{\circ} \mathrm{C}$ (Fig.2). That is, at the deposition temperature of $410{ }^{\circ} \mathrm{C}$, an epitaxial InSb film is formed. At the deposition temperature of $420{ }^{\circ} \mathrm{C}$, the degree of epitaxy of the InSb film decreases, as additional diffraction reflections of significant intensity appear: (020) and (313) (Fig. 4). Scanning-electronic images of the orientation of the InSb film crystallites show the formation of non-directional (100) crystallites in the film (Fig. 5). A small amount of the In phase is observed due to the vacuum sublimation of $\mathrm{Sb}$ from InSb. It is assumed that in the film the smaller crystallites with (100) orientation combine into larger ones. 


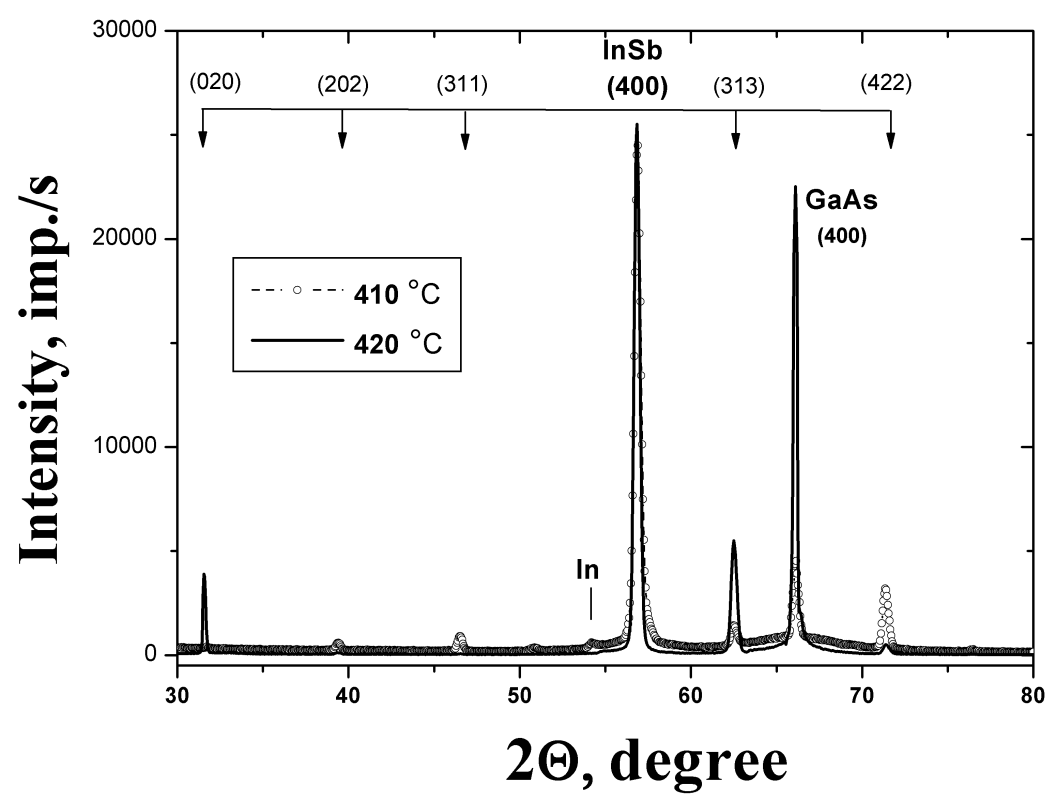

Figure 4. XRD patterns from film samples deposited on semi-insulating GaAs plates by explosive thermal evaporation of InSb powder at temperatures $410^{\circ} \mathrm{C}$ and $420^{\circ} \mathrm{C}$

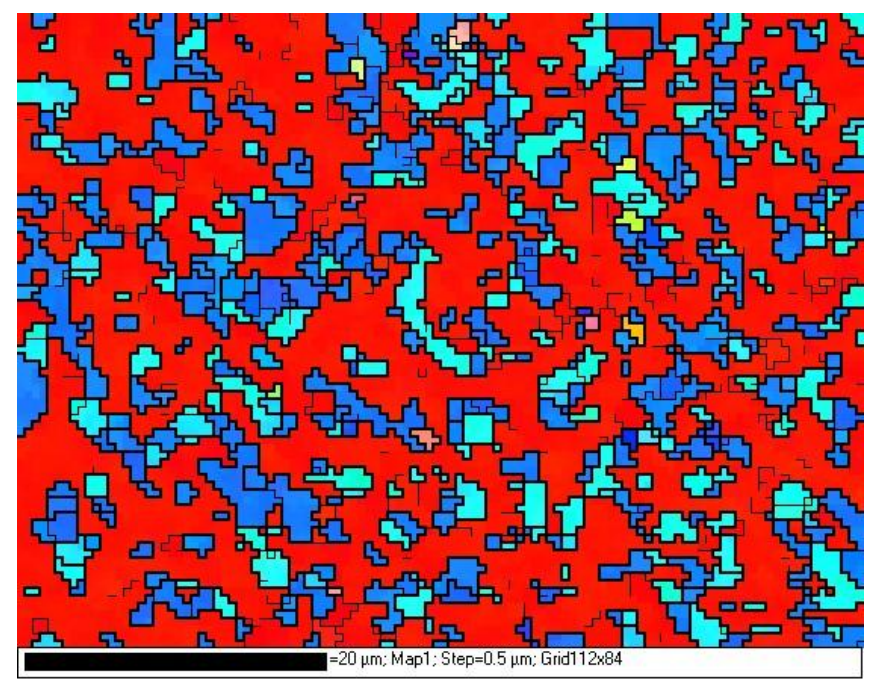

Figure 5. Scanning images of the orientation of crystallites of the InSb film deposited at temperature of $420{ }^{\circ} \mathrm{C}$

Scanning-electronic images of the surface of InSb films at deposition temperatures of 375 and $420^{\circ} \mathrm{C}$ are displayed in Fig. 6. It is seen that the surface roughness of the film increases with 
an increase in temperature to $420^{\circ} \mathrm{C}$. The increase in the film surface roughness is confirmed by the formation of larger InSb film crystallites.

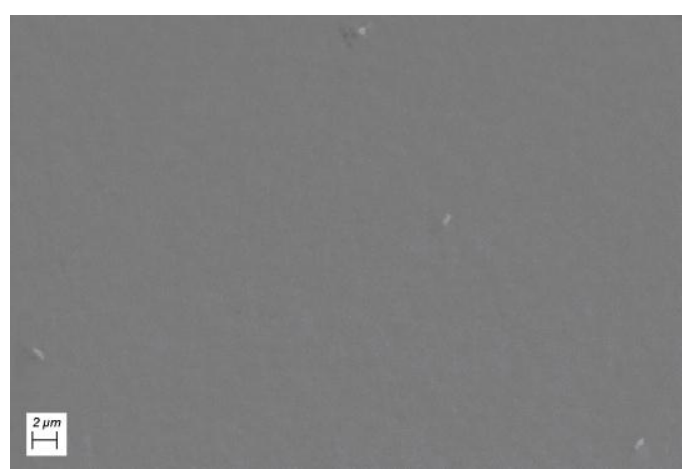

a

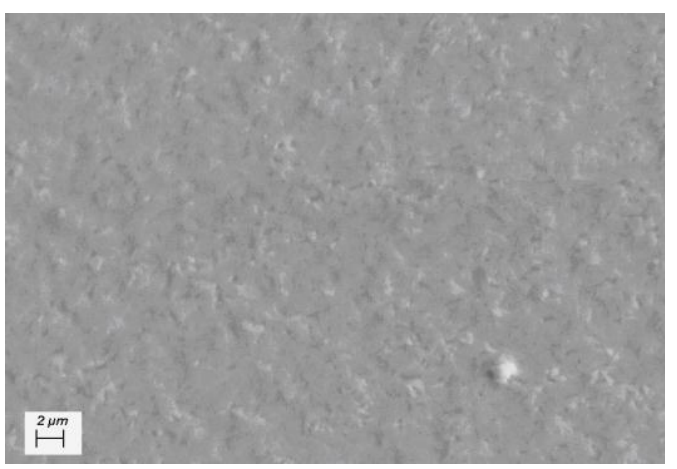

b

Figure 6.Scanning electronic images of the surface of InSb films obtained by explosive thermal evaporation at deposition temperatures: $a-375^{\circ} \mathrm{C}, 6-420^{\circ} \mathrm{C}$

From the analysis of the data presented above (Figs. 2, 3c, 4, 6a), it follows that during the explosive thermal deposition of InSb on semi-insulating GaAs (100) plates at temperatures of $375-410^{\circ} \mathrm{C}$, heteroepitaxial InSb films form with a preferred crystallite orientation (100).

Electric and magnetic properties of InSb films on GaAs formed by explosive thermal deposition at various temperatures are presented in the table.

Table. Characteristics of InSb films on GaAs depending on the deposition temperature

\begin{tabular}{|c|c|c|c|c|c|}
\hline $\begin{array}{c}\text { Deposition temperature, } \\
{ }^{\circ} \mathrm{C}\end{array}$ & $\begin{array}{c}\text { Thickness, } \\
\mu \mathrm{m}\end{array}$ & $\mathrm{R}, \Omega$ & $\mathrm{U}_{\mathrm{x}}, \mathrm{V}$ & $\mathrm{n}, 1 / \mathrm{cm}^{3}$ & $\mu, \mathrm{cm}^{2} /(\mathrm{V} \times \mathrm{s})$ \\
\hline 350 & 1.1 & 56 & 0.25 & $(1.44 \pm 0.08) \times 10^{17}$ & $(4.5 \pm 0.4) \times 10^{3}$ \\
\hline 366 & 3.2 & 45 & 0.51 & $(1.61 \pm 0.09) \times 10^{16}$ & $(5.1 \pm 0.5) \times 10^{3}$ \\
\hline 375 & 2.6 & 10 & 0.65 & $(2.2 \pm 0.1) \times 10^{16}$ & $(18.8 \pm 1.8) \times 10^{3}$ \\
\hline 410 & 3.0 & 17 & 0.41 & $(6.5 \pm 0.4) \times 10^{16}$ & $(15.9 \pm 1.6) \times 10^{3}$ \\
\hline 420 & 3.0 & 33 & 0.13 & $(2.9 \pm 0.2) \times 10^{16}$ & $(5.9 \pm 0.6) \times 10^{3}$ \\
\hline
\end{tabular}


The data in the table show that InSb films on GaAs have high Hall voltage values in the deposition temperature range of $375-410{ }^{\circ} \mathrm{C}$. The maximum values of the Hall mobility of charge carriers reach values up to $(18.8 \pm 1.8) \times 10^{3} \mathrm{~cm}^{2} /(\mathrm{V} \times \mathrm{s})$. The high charge carrier concentrations obtained at the deposition temperature of $350{ }^{\circ} \mathrm{C}$ compared to films deposited at other temperatures are due to the lower thickness of the deposited film. As the film thickness decreases, the defect transition layer containing many dislocations between the substrate and the growing film crystals, associating with a large discrepancy between the lattice parameter of GaAs and InSb, influences the increase in the carrier concentration (Vishwakarma et al., 2013). It is known from the literature that the maximum values of the Hall mobility of charge carriers during the deposition of InSb films on GaAs by MBE are achieved when the film thickness is at least $2 \mu \mathrm{m}$ and the deposition temperature is from $300{ }^{\circ} \mathrm{C}$ (the first thin layer) to $395{ }^{\circ} \mathrm{C}$ (the final main layer) (Debnath et al., 2004). The deposition temperature range of $375-410{ }^{\circ} \mathrm{C}$ during explosive thermal deposition corresponds to the high electrical characteristics of the obtained films in this work largely corresponds to the temperature conditions for the deposition of perfect films obtained by MBE.

The usage of method of explosive thermal evaporation has shown the following advantages over MBE: It does not require sophisticated equipment to create molecular beams of controlled density and homogeneity; high repeatability of results in terms of carrier concentration and mobility, homogeneity and phase composition of the grown films; wide range of thicknesses from 0.5 to $10 \mu \mathrm{m}$ and concentrations of grown epitaxial structures.

\section{Conclusions}


The XRD and EBSD investigations of the structure and orientation of the crystallites of InSb films as a function of temperature of deposition by explosive thermal evaporation on GaAs (100) substrates have shown that high-quality heteroepitaxial InSb films are formed at deposition temperature range of $375-410{ }^{\circ} \mathrm{C}$. At lower deposition temperatures, InSb films have a polycrystalline structure. At a deposition temperature of $420{ }^{\circ} \mathrm{C}$, the film has a predominant GaAs substrate orientation, but the degree of epitaxial perfection of the film decreases due to the presence of other orientations of InSb crystallites. The obtained heteroepitaxial InSb films have high values of the Hall potential (more than $0.5-0.6 \mathrm{~V}$ ), the values of the Hall mobility of the charge carriers are in the range of $(16-19) \times 10^{3} \mathrm{~cm}^{2} /(\mathrm{V} \times \mathrm{s})$. The electrical properties of the formed heteroepitaxial InSb films on GaAs allow them to be used as highly sensitive miniature Hall convertor.

\section{References}

Balvanovich E. I., Semiconductor films and miniature measuring transducers, Minsk: Naukaitekhnika, 1981. (in Russian)

Bennett B. R., Magno R., Boos J. B., Kruppa W., Ancona M. G., Antimonide-Based Compound Semiconductors for Electronic Devices: A Review. Solid-State Electronics. Vol. 49, pp.1875-1895, 2005.

Brent L. A. et.al. The method of diffraction of reflected electrons in materials science. S. A. Ivanov, Trans. Moscow: Technosphere, 2014. (in Russian)

Debnath M.C., Zhang T., Roberts C., Cohen L.F., Stradling R.A., High-mobility InSb thin films on GaAs (001) substrate grown by the two-step growth process. Journal of Crystal Growth. Vol. 267, pp. 17-21, 2004. 
Joyce B.A., Hackingbottom R., Menh W. et al. Molecular beam epitaxy and heterostructures. Zh. I. Alferov, Yu. V. Shmartsev, Trans. Moscow: Mir, 1989. (in Russian)

Kimukin I., Biyikli N., Ozbay E., InSb high-speed photodetectors grown on GaAs substrate. Journal of applied physics. Vol. 94, pp. 5414-5416, 2003.

Kuchis E. V., Methods of studying the Hall effect, Moscow: Sov. radio, 1974. (in Russian)

Maissel L., Glang R., Thin film technology. Guide. Vol. 1, Moscow: Sov.radio, 1977. (in Russian)

Okamoto A., Shibasaki I., Transport properties of Sn-doped InSb thin films and applications to Hall element. Journal of Crystal Growth. Vol. 251, pp. 560-564, 2003.

Oszwaldowskia M., Berus T., Hall sensors made of $\mathrm{n}-\mathrm{InSb} / \mathrm{GaAs}$ epitaxial layers for low temperature applications. Thin Solid Films. Vol. 515, pp. 2692-2695, 2006.

Oszwaldowskia M., Berus T., Borowskaa A., Nowickia M., Richterb A., Sangwal K., Textural properties of InSb thin films. Journal of Crystal Growth. Vol. 265, pp. 83-93, 2004.

Rahul, Tripathi R.S.N., Kumar A., Vishwakarma S.R., Electrical characterization of electron beam evaporated Indium Antimonide thin films. Archives of Physics Research. Vol. 2 (2), pp. 100-106, 2011.

Vishwakarma S.R., Kumar A., Tripathi R.S.N., Rahul, Dus S., Fabrication and characterization of $\mathrm{n}$-InSb thin film of different thicknesses. Indian Journal of Pure \& Applied Physics. Vol. 51, pp. 260-266, 2013.

Zhang T., Clowes S. K., Debnath M., Bennett A., Roberts C., Harris J. J., Stradling R. A., High-mobility thin InSb films grown by molecular beam epitaxy. Applied Physics Letters. Vol. 84 (22), pp. 4463-4465, 2004. 